\title{
Energy performance assessment of thermo-active micro-piles via numerical modeling and statistical analysis
}

\author{
Francesco Cecinato $^{1}$ and Diana Salciarini ${ }^{2}$ \\ ${ }^{1}$ Dipartimento di Scienze della Terra “A. Desio”, Università degli Studi di Milano \\ Via Mangiagalli, 34, 20133 Milan (Italy) \\ francesco.cecinato@unimi.it \\ ${ }^{2}$ Department of Civil and Environmental Engineering, Università degli Studi di Perugia \\ via G. Duranti 93, 06125, Perugia (Italy) \\ diana.salciarini@unipg.it
}

\begin{abstract}
Energy micro-piles (EMPs) constitute a promising emerging technology, able to provide both energy and structural retrofitting to existing buildings. Although a number of studies have been published on the energy performance of standard energy piles (EPs), bespoke analysis is required for EMPs due to their different geometry and peculiar design and site constraints. In this work, a parametric study is carried out by means of numerical Finite Element simulations complemented by statistical analysis, aimed at identifying the dominant parameters in maximizing EMP thermal efficiency. Two categories of parameters, namely design-dependent and site-dependent ones, are separately considered to provide guidance for practitioners on both detailed geothermal sizing and overall feasibility assessment. The parameter space is efficiently explored resorting to Taguchi Experimental Design statistical tools. Results show that different design criteria to those for EPs should be used for EMPs. Notably for the latter, contrary to the former, the diameter of heat exchanger pipes emerges as one of the most important factors promoting thermal efficiency, while being the single easiest parameter to engineer. On the other hand, while maximizing the number of U-pipes and pile diameter is crucial for EPs, it does not impact the energy performance of EMPs, due to geometry constraints and the expected occurrence of thermal interferences. Among site-dependent factors, a large ground thermal conductivity is confirmed as an important feature to ensure a high energy performance, while interesting insights are obtained about the role of basement thermal insulation in the long-term EMP thermal output.
\end{abstract}

Keywords: energy foundations, numerical modeling, micropiles, shallow geothermal energy, retrofitting 


\section{Introduction}

Energy Geo-Structures (EGS) represent an innovative and multifunctional technology that can be used for energy applications as well as to provide structural support to any type of built environment [1]. Similarly to other traditional geothermal systems, EGS exploit low-enthalpy geothermal energy, based on the fundamental property of the first tens to a few hundreds of meters of subsoil of keeping a virtually constant temperature throughout the year. EGS can serve the purposes of several energy applications, such as (1) incorporating the primary circuit of space heating and cooling systems, typically coupled to a ground source heat pump (GSHP), (2) contributing to the production of warm water for domestic, agricultural or industrial uses, (3) providing heat to prevent the formation of ice on sidewalks, bridge decks, roads and airport runways and (4) storing heat in the subsoil for subsequent use.

To date, numerous literature studies have been devoted to modeling both the thermal and thermo-mechanical response of EGS during both monotonous and cyclic extraction/injection of heat from/into the ground, considering various technologies including: Energy Piles (EPs) (e.g., [2]-[10]), energy tunnels (e.g., [11]-[14]), and energy diaphragm walls (e.g., [7], [15]-[17]). A series of field tests, small-scale laboratory tests and physical model tests on EPs to understand their ThermoHydro-Mechanical (THM) behavior have been also carried out. Existing literature refers either to the behavior of a single EP or to groups of EPs, and the corresponding thermally induced group effects ([3], [8], [18], [19]). Also, a number of pilot field applications has been implemented in Europe in the last two decades. Significant examples include the foundations of terminal $\mathrm{E}$ of Zurich airport $(\mathrm{CH})$, with a total of 315 thermo-active piles out of 440 [20]; the foundations of Lambeth College in London (UK), with 143 EPs [4]; and the World Expo Axis building in Shanghai (China), with approximately 6000 EPs [21].

EPs are most typically installed with cast-in-place (e.g. rotary bored or Continuous Flight Auger) technology, but also precast driven EPs have been recently proposed (e.g. [22]). In all cases, EPs have so far always been employed as the foundation system of newly constructed buildings. An attractive possibility, that would extend the field of application of EGS to building renovation and reinforcement projects, is to use EPs of small diameter (less than $30 \mathrm{~cm}$ ), so-called Energy Micro-Piles (EMPs). In traditional applications, micro-piles are typically used in underpinning projects for existing structures that need structural retrofitting, to increase the bearing capacity of the soil-foundation system and/or reduce settlements (e.g., [23], [24]). Depending on the heating and cooling requirements of the building, all or a subset of the micropiles used for structural/geotechnical purposes can be equipped with heat exchangers, so as to combine their structural role with an energetic one. In other words, EMPs can provide both structural and energy retrofitting to existing buildings. This opportunity is even more timely and significant in light of the recently released public incentive measures to promote both energy and structural/seismic retrofitting of existing buildings, in certain European countries such as Italy (e.g. see [25]). 
On the other hand, micro-piles could be employed also in new construction projects, whenever the geotechnical conditions or site constraints (e.g., the lack of space for standard piling equipment, which may be the case in highly inhabited historic town contexts) makes them a convenient solution. Also in this case, adopting EMPs may bring about advantages such as cost savings compared to traditional shallow geothermal solutions (i.e., EGS generally remove the need to make special purpose excavations), as well as the possibility to engineer some thermal parameters, as will be discussed below (Section 3.1.2).

While research on EPs started in the late 1990s and was very prolific, there is very little literature research focused on EMPs. Ronchi et al. [26], [27] presented the results of field test experimental activities and related numerical simulations for a prototype of EMP. They showed that the value of specific heat flux generated by the test EMP falls within the same range of that of conventional EPs and that the EMP performance is mainly affected by the thermal properties of the foundation soil. Lautkankare et al. [28] investigated technical issues in the utilization of EMPs in underpinning projects, related to the through holes for geothermal energy collector pipes in the load transfer structures. The Authors presented nine possible technical solutions along with construction details and recommendations for the application of EMPs to existing buildings. Kong et al. [21] presented a first EMP installation in China where an existing piled raft foundation on silty clay was underpinned with 8 micro-piles, two of which equipped with heat exchangers. Results showed that the EMP-raft foundation could provide adequate heat exchange compared with other types of ground heat exchangers. Differential settlements at both the pile top and tip were observed for the groups that contained both EMP and standard piles. Tyszer \& Tomaszewska [29] investigated the possibility of using traditional commercial micro-piles as heat exchangers in Poland, concluding that this application in most parts of the country would be cost-effective and 50\% more efficient compared to adopting conventional heat energy acquisition technologies. Ren et al. [30] presented results from field tests to assess the thermal response of micro-steel-pipe piles under temperature cycling, observing that heat transfer efficiency was different between winter and summer conditions, and it decreased with increasing number of cycles.

A number of previous works demonstrated that several parameters affect the thermal performance of EGS. These may be subdivided into two subgroups: i) design parameters, i.e. those that could be chosen by the engineer during dimensioning of the system (including: configuration, geometry, materials, operating temperature, flow rate, fluid thermal properties, e.g. see [5]); ii) site parameters, i.e. those concerning the site/environmental conditions that are independent of the project (e.g., see [31]), but should be taken into account to ensure adequate system design (including: soil thermal properties, existing connections with the superstructure, external seasonal temperature).

In recent years, a number of authors presented studies on EPs focused on understanding the effects of some of the abovementioned parameters. Batini et al. [2] presented the results of numerical sensitivity analyses on the thermo-mechanical response of a full-scale EP for different pipe configurations, foundation aspect ratios, mass flow rates of the heat carrier 
fluid and fluid mixture compositions. Cecinato \& Loveridge [5] considered the influences on the thermal efficiency of EPs of design parameters such as the number of pipes, fluid flowrate, pile geometry and concrete conductivity, by means of a FEM numerical model used to perform a Taguchi parametric analysis. Bezyan et al. [32] compared, via 3D heat transfer simulations, the performance of EPs with Spiral shaped, U-shaped and W-shaped heat exchanger pipes. Yang et al. [33] described experimental testing of the thermal performance of an EP with spiral coil, investigating the influences of inlet water temperature, intermittent operation mode, spiral pitch and pile material. Similarly, Carotenuto et al. [34] presented an assessment of EP heat transfer performance considering different pipes and pile diameters, material properties, fluid flow rate and pipe configurations (U-tube, Double U-tubes, Triple U-tubes, spiral coil). Salciarini et al. [9] presented a parametric study on the THM response of a large piled raft equipped with EPS, varying the soil's thermal expansion coefficient, thermal conductivity and the EP layout. Fadejev et al. [35] presented a review on EP design, with different fundamental schemes of heat pump systems, and various EP configuration types. Park et al. [36] produced an engineering chart for the thermal performance of cast-in-place energy pile considering various layouts of heat exchanger pipes. Sangwo et al. [37] studied the effect of thermal interference on energy piles considering various configurations of heat exchangers.

Despite the numerous attempts in previous literature to explore the influence of some of the influential design and site parameters in the performance of EPs, a comprehensive study has never been published that considers in a systematic manner all of the relevant parameters to provide a rating of their impact on the energy response of EMPs.

In this work, the influence of both design and site parameters on the EMP thermal performance is investigated numerically, in terms of exchanged thermal power. To this aim, reference is made to the EMP prototype developed and installed at the Engineering Campus of the University of Perugia, building up on the parametric analyses carried out by Ronchi et al. [26] and Salciarini \& Cecinato [38]. To more efficiently explore parameter space, the parametric studies are performed using the Taguchi statistical approach, considering ranges of variability for the involved parameters derived from real case studies. The aim of this study is twofold: on the one hand, the most dominant parameters on the EMP thermal behavior are highlighted, and on the other hand, the optimal parameter value combinations to maximize EMP performance are identified, to provide design guidance to practitioners. The rationale of the work is as follows: first, the dominant parameters are identified among design-dependent ones (set \#1, namely: fluid velocity, pipe material thermal conductivity, fluid thermal conductivity, pile diameter, pile length, concrete thermal conductivity, pipe diameter). Secondly, with reference to the best parameter combination obtained with the set \#1 analysis, a parametric study is conducted considering the influence of site-dependent parameters (set \#2, namely: soil conductivity, soil specific heat capacity, thickness of insulating layer, environment temperature). 
The remainder of the paper is structured as follows. In Section 2 the theoretical formulation and the numerical model settings are outlined. In Section 3.1 the choice of relevant parameters and corresponding ranges is discussed, while Section 3.2 presents the design of the two Taguchi parametric analyses. In Section 3.3 the impact of both parameter sets in the performance of EMPs as a result of the parametric study is discussed, and conclusions are drawn in Section 4.

\section{Model formulation}

\subsection{Governing equations}

The heat exchange processes taking place in the pipe-pile-soil system are: a) convective heat exchange between heatcarrier fluid and pipes walls; b) heat conduction in the pile grout; c) heat conduction in the soil domain. The heat conduction in continuous media (i.e., the grout and the soil) is described by the classical transient heat conduction equation (e.g., see [39]):

$$
\rho c_{P} \frac{\partial T}{\partial t}=-\nabla \boldsymbol{q}
$$

where $\boldsymbol{q}=\lambda \nabla T$ is the heat flux vector, $\lambda$ the thermal conductivity of the material and $T$ the temperature, $\rho$ is the material density, $c_{P}$ is the specific heat at constant pressure. The non-isothermal flow in the pipes can be formulated as a $1 \mathrm{D}$ problem, given the large difference in scale between the pipe diameter and the other dimensions of the problem. Thus, modeling the pipes as linear elements, the governing equations are obtained by the momentum conservation equation:

$$
\rho_{f} \frac{\partial \boldsymbol{u}}{\partial t}=-\nabla p-f_{D} \frac{\rho_{f}}{2 d_{h}}|\boldsymbol{u}| \boldsymbol{u}+\boldsymbol{F}
$$

and the mass conservation equation:

$$
\frac{\partial A_{p} \rho_{f}}{\partial t}+\nabla\left(A_{\boldsymbol{p}} \rho_{f} \boldsymbol{u}\right)=0
$$

where $\rho_{f}$ is the fluid density; $\boldsymbol{u}$ the cross section averaged fluid velocity; $p$ the fluid pressure; $f_{D}$ the Darcy friction factor that can be obtained as a function of the Reynolds number (see, e.g., [40]); $\boldsymbol{F}$ is a body force density; $A_{\boldsymbol{p}}$ is the pipe cross section area, and $d_{h}$ is the mean hydraulic diameter. For an incompressible fluid circulating within the pipes, the energy balance equation is given by:

$A_{p} \rho_{f} c_{p f} \frac{\partial T}{\partial t}+A_{p} \rho_{f} c_{p f} \mathbf{u} \cdot \nabla \mathrm{T}=\nabla A_{p} \lambda_{f} \nabla \mathrm{T}+f_{D} \frac{\rho_{f} A_{p}}{2 d_{h}}|\boldsymbol{u}|^{3}+q^{\prime}{ }_{\text {wall }}$ 
where $c_{p f}$ is the specific heat of the fluid and $\lambda_{f}$ the fluid thermal conductivity. The second term on the right-hand side of eq. (4) represents the heat dissipated by internal friction in the fluid, while $q_{\text {wall }}^{\prime}$ accounts for heat exchanged radially between the pipe and the surrounding continuous media.

\subsection{Numerical model}

The considered thermo-active structure is a cast-in-place micropile, with tubular steel reinforcement, equipped with heat exchanger pipes representing the primary circuit of a GSHP system. Following Ronchi et al. [26], reference is made to an existing prototype of energy micropile, designed with the aim of improving the geostructure's heat exchange capacity compared to standard energy piles, by achieving a more homogeneous temperature field in the pile toe area (corresponding to the deepest portion of the surrounding soil, that is also the least affected one by surface seasonal temperature oscillations). This was obtained by equipping the pile with a so-called 'energy tip', i.e., a 6-liter capacity steel tank located at the bottom of the pile, filled with heat-carrier fluid and connected in series to the pipes, thus completing the U-loop shape of the fluid circulating system.

A three-dimensional Finite Element (FE) model of such thermo-active geostructure installed within a fine-grained soil domain was created using the Comsol Multiphysics code, to gain insights on the energy performance of the micropile during geothermal operation.

A cylindrical domain of $20 \mathrm{~m}$ diameter and $30 \mathrm{~m}$ height was considered, with a single energy micropile (EMP) placed around its axis of symmetry. The size of the domain was chosen by numerical experimentation to be significantly larger than the area actually affected by heat transfer, for the time ranges explored in this work. The fluid flow and convective heat transfer within the pipes were modeled in terms of section-averaged quantities, while fully modeling the surrounding domain as a $3 \mathrm{D}$ continuum.

As described in Section 2.1, only heat conduction is assumed to take place in the soil, grout/concrete and steel materials, implying that additional thermal phenomena, such as thermal radiation at the soil surface and convective heat transfer in the pore water, are neglected. While the former phenomenon is typically considered negligible in all but the coarsest of soils (e.g. [41], [42]), the possible importance of groundwater convection restricts the applicability of the current model to cases of low-permeability or dry soils and rocks. However, the model can be readily extended to account for groundwater advection whenever relevant.

The saturated soil material was thus represented by solid elements, although its bulk thermo-physical properties were assigned by accounting for the different properties of pore water and the solid grains, by appropriately considering the solid and voids fractions. For example, the specific heat capacity of the ground was deduced as $c_{p g}=n c_{p w}+(1-n) c_{p s}$, 
where $n$ the porosity, $c_{p w}$ the specific heat capacity of water at ambient temperature and $c_{p s}$ the specific heat capacity of soil particles.

For ease of representation, the water tank at the energy tip was represented as a continuous solid volume crossed by the 1D pipe elements, with the thermal properties of water (Figure 1a). A view of the modeled domain, along with the spatial discretization adopted, is shown in Figure $1 \mathrm{~b}$.

The interested reader is referred to [26] for further details on the basic settings of the EMP FE model, including its validation against experimental data, consisting of numerically reproducing the experimental measurements of outlet temperature histories collected during a bespoke Thermal Response Test.

In the first parametric study discussed in Section 3.1.1, focused on assessing the influence of typical micropile design parameters (in other terms, parameters that can be engineered) in their energy efficiency, the bulk of numerical settings described above and in [26] was adopted. In particular, a constant fluid inlet temperature of $35^{\circ} \mathrm{C}$ was adopted, to emphasize the effect of design factors on the EMP thermal output, while adopting a constant temperature boundary condition (equal to the undisturbed soil temperature, $16^{\circ} \mathrm{C}$ ) at all domain borders. However, both pipe and pile diameter and pile length were allowed to change, to accommodate typical micropile and pipe geometry ranges.
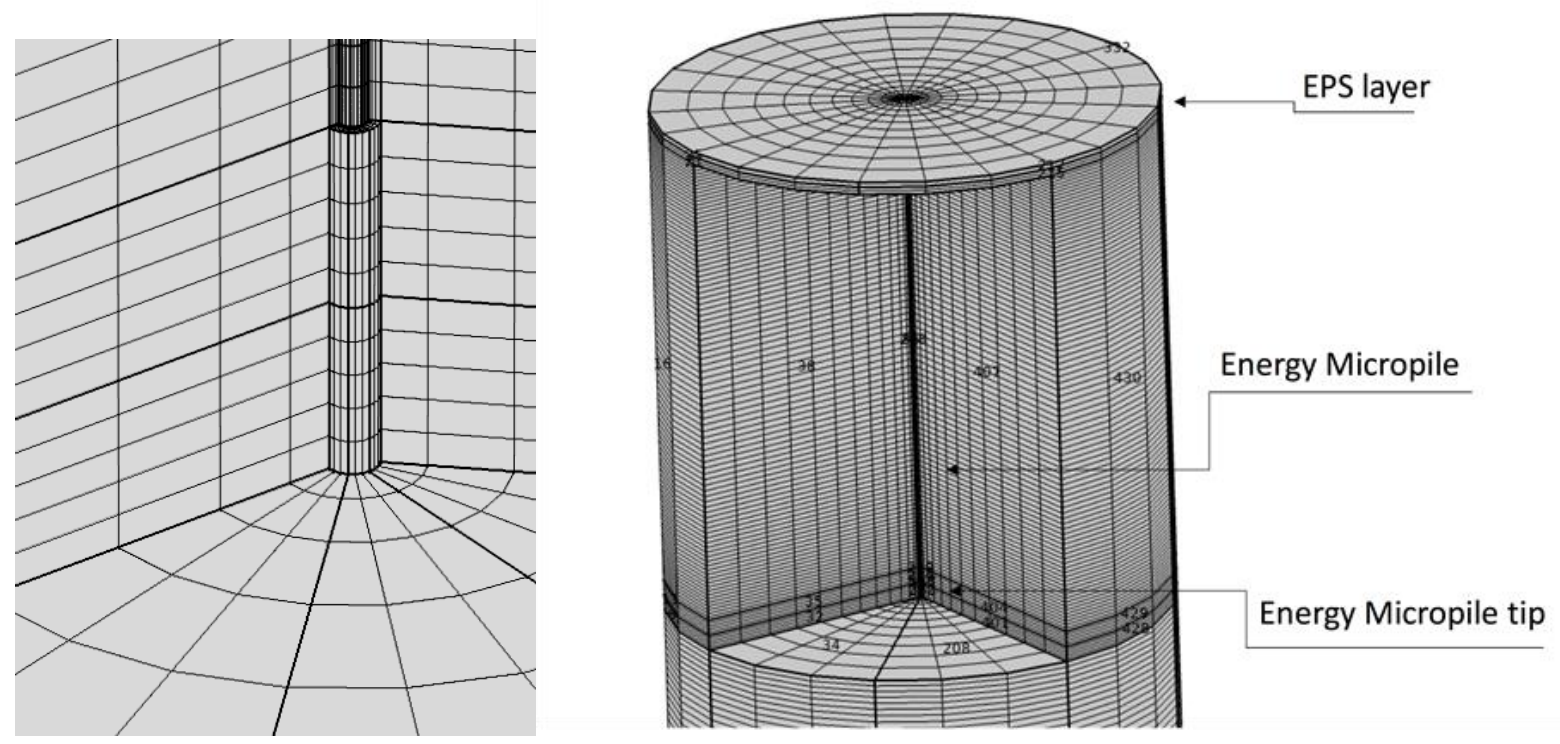

Figure 1. Details of the FE model geometry and discretization. a) Energy tip, b) domain spatial discretization with geometry details.

In the second parametric analysis (see Section 3.1.2), to be able to investigate the thermal consequences of varying site conditions (in other terms, parameters that depend on the pre-existing situation and cannot be engineered), the numerical model of Ronchi et al. [26] was further modified, by generalizing both the geometry and boundary conditions at the top 
of the domain, and the initial conditions. Specifically, the geometry was modified by allowing for the presence of two additional layers above the pile head and soil surface level (Figure 1b): (1) the pile head was overlain by a $30 \mathrm{~cm}$ thick concrete layer that represents a concrete slab, but may also be considered to thermally represent a generic structure that is supported by the EMP, such as a plinth or other shallow foundation structure; (2) an expanded polystyrene (EPS) layer, of variable thickness within a reasonable range (see Section 3.1.2), was placed above the concrete layer, representing typical thermally insulating panels that are employed in new buildings to improve their energy efficiency (e.g., see [43]). To obtain more realistic operational conditions, boundary and initial conditions were improved compared to the existing model of [26]. Boundary conditions were set as adiabatic for the bottom and lateral surface of the domain, and equal to a fixed temperature for the domain top surface, representing the average temperature of air in contact with the external surface of the foundation slab/plinth. As regards initial conditions, the initial temperature in the solid domain was obtained upon running a 'thermal initialization' simulation, i.e. a transient heat conduction simulation (while disabling any fluid movement and convective heat exchange within the pipes) for 180 days, starting from an initial homogeneous temperature condition $\mathrm{T}_{0}=16^{\circ} \mathrm{C}$, and keeping a constant boundary temperature at the top of the concrete slab (in the absence of the overlying EPS insulating layer) equal to $23^{\circ} \mathrm{C}$, representing the average warm-season air temperature in the Italian/Southern European area (e.g., see [44]). Although the real boundary temperature will exhibit natural fluctuations over a 180day period, a fixed average temperature value was chosen (i) for the sake of simplicity and (ii) for ease of comparison between short- and long-term results. The resulting temperature profile (Figure 2) can be considered representative of natural underground conditions in the warm season, including the surficial deviation from thermal homogeneity due to the atmospheric temperature influence. Starting from a realistic soil temperature profile is deemed especially important for EMPs, whose real thermal behavior is expected to be influenced by surface effects, being their length comparatively shorter than other deep foundations.

After thermal initialization, GSHP operation was simulated by activating non-isothermal flow within the pipes, with a constant inlet fluid temperature of $31^{\circ} \mathrm{C}$. This corresponds to imposing a maximum $\Delta \mathrm{T}=15^{\circ} \mathrm{C}$ compared to the undisturbed ground temperature, and is consistent with usual upper-bound values of the imposed $\Delta \mathrm{T}$ for shallow geothermal applications (e.g., typical operational values of $\Delta \mathrm{T}$ around $8^{\circ} \mathrm{C}$ are suggested by [2], [5], [7], while [45] adopt an upperbound value of $\Delta \mathrm{T}=15^{\circ} \mathrm{C}$ to assess thermo-mechanical couplings in energy piles). Adopting a larger than average (yet reasonable) value of $\Delta \mathrm{T}$ brings about the advantage of emphasizing the relative importance of different parameters in enhancing thermal performance, rather than quantifying the energy performance in absolute terms (e.g. in terms of specific heat flux per unit length of the pile). It should be observed that real systems usually operate within varying thermal demand patterns. However, a constant inlet temperature was used in this work to achieve 1) a simpler comparison of the parameters under consideration and 2) a more general approach, to embrace the wide range of possible thermal demand 
scenarios. In this case heat injection only has been applied, representing summertime operation, reflecting the expected predominant use of EMPs in Southern Europe, also in view of forthcoming climate change/global warming effects. It can be also observed that in the absence of any 'thermal drift' effects, and in the presence of a perfectly balanced scenario between summer and winter thermal loads, if the temperature differences (in absolute value) between the inlet temperature and the initial ground temperature at all depths are the same, the relative importance of the different parameters considered in Section 3.1 in the EMP thermal performance is expected to remain the same, regardless of the direction of heat transfer.
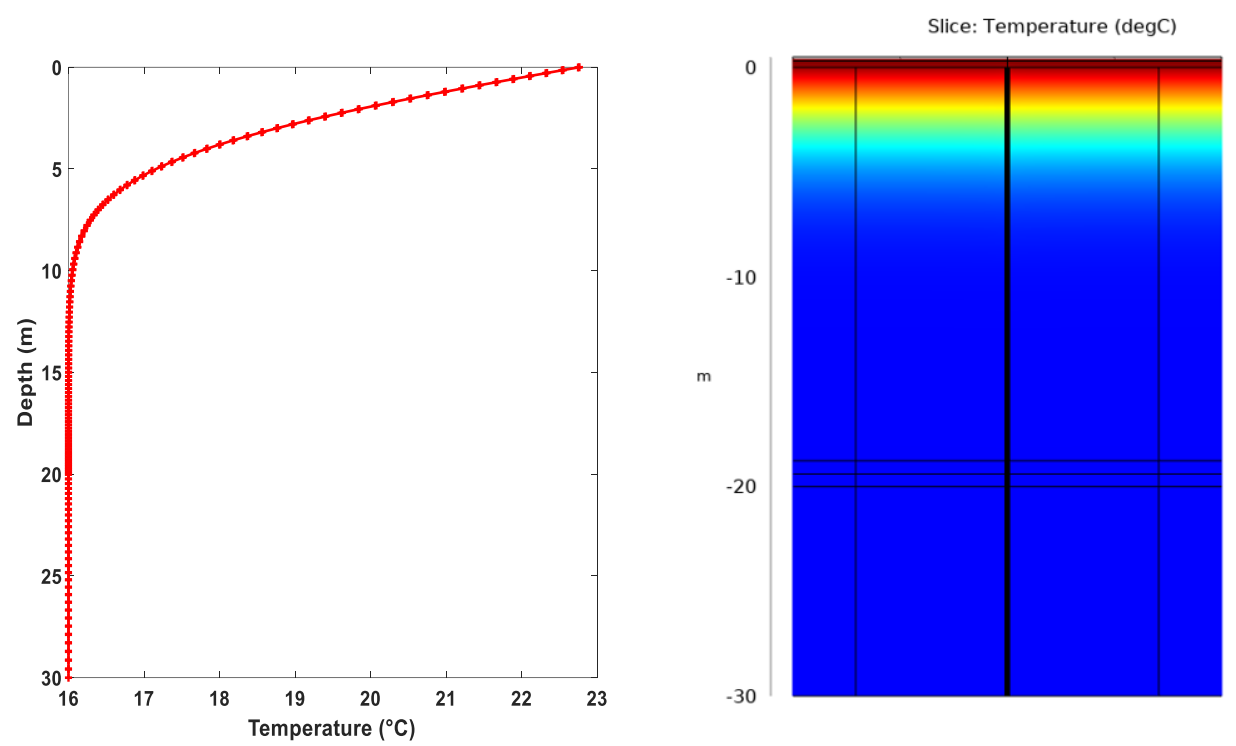

Figure 2. Initial temperature profile within the ground domain as a result of thermal initialization simulation, (a) $1 D$ profile taken along a vertical line located $0.5 \mathrm{~m}$ from the pile center, (b) temperature contour lines in a longitudinal cross-section of the domain

\section{Energy efficiency of EMPs}

Assessing the influences on the thermal efficiency of EMPs is mainly aimed at establishing design criteria that may aid practicing engineers to take decisions on thermal (and related geotechnical) aspects of the geothermal foundation project. Regardless of whether thermo-active micropiles are to be applied to a pre-existing building (hence, in the frame of a both thermal and structural retrofitting project) or a new one (whenever the micropile technology is deemed advantageous over other foundation types), their overall design will depend on two distinct categories of parameters: i) proper design parameters (i.e. those that may be engineered), and ii) construction site parameters (i.e. those that depend on the field's pre-existing situation and cannot be engineered). Typically, the former relate to the EMP geometry, materials and other design settings, while the latter concern the thermo-physical properties of the ground and any pre-existing structure interacting with the EMP. 
Based on the above distinction, two separate numerical parametric studies are presented in this section, to maximize the thermal performance of EMPs. The first one is aimed at the optimization of design parameters (hereafter referred to as 'set $\# 1$ '), the second one is intended for the overall evaluation of the convenience of the EMP solution by identifying the most influential site properties (hereafter referred to as 'set \#2').

\subsection{Choice of parameters and relevant ranges}

In this subsection, the choice of the design-dependent and site-dependent parameter sets and their relevant ranges are discussed, based on available literature information and the experience gained from existing engineering projects and previous works carried out by the authors on energy piles.

\subsubsection{Design dependent parameters}

Design dependent parameters were chosen, among all possible model parameters, as the ones that are potentially easiest to engineer. These are summarized in Table 1.

Table 1. Set \#1 of design dependent parameters and relevant ranges of variation.

\begin{tabular}{l|c|c|c|c|c|c|c} 
Parameters & $\begin{array}{c}\text { Fluid } \\
\text { velocity } \\
v\end{array}$ & $\begin{array}{c}\text { Pipe } \\
\text { material } \\
\text { conductivity } \\
\lambda_{p}\end{array}$ & $\begin{array}{c}\text { Thermal- } \\
\text { carrier fluid } \\
\text { conductivity } \\
\lambda_{f}\end{array}$ & $\begin{array}{c}\text { Micropile } \\
\text { diameter } \\
D_{\text {pile }}\end{array}$ & $\begin{array}{c}\text { Micropile } \\
\text { length } \\
L\end{array}$ & $\begin{array}{c}\text { Concrete } \\
\text { thermal } \\
\text { conductivity } \\
\lambda_{c}\end{array}$ & $\begin{array}{c}\text { Pipe } \\
\text { diameter } \\
d_{\text {pipe }}\end{array}$ \\
\hline Units & $\mathrm{m} / \mathrm{s}$ & $\mathrm{W} /(\mathrm{m} \cdot \mathrm{K})$ & $\mathrm{W} /(\mathrm{m} \cdot \mathrm{K})$ & $\mathrm{mm}$ & $\mathrm{m}$ & $\mathrm{W} /(\mathrm{m} \cdot \mathrm{K})$ & $\mathrm{mm}$ \\
\hline Lower bound (MIN) & 0.4 & 0.6 & 0.6 & 150 & 10 & 1.5 & 12 \\
\hline Upper bound (MAX) & 1.2 & 20 & 0.79 & 200 & 20 & 3 & 20 \\
\hline
\end{tabular}

As regards the micropile object, a designer can in principle change its geometry and construction materials, to a certain extent, subordinate to geotechnical and structural criteria as well as construction/factory constraints. To this end, the pile diameter $D_{\text {pile }}$, the pile length $L$ and the concrete thermal conductivity $\lambda_{c}$ were selected as representative variables. $D_{\text {pile }}$ was set to vary between 10 and $20 \mathrm{~cm}$, since this range can be considered typical of the most widespread micropiles as indicated in established textbooks and design manuals (e.g., see [23], [24]). Along the same lines, $L$ was set to range between 10 and $20 \mathrm{~m}$ (e.g., [46]). Parameter $\lambda_{c}$ is not deemed to be as easily engineered as the pile geometry. However, previous studies suggest [47], [48] that while $\lambda_{c}$ depends on the aggregate lithology, which in practice is determined by the locally available materials for economic and ecological reasons, its overall value can be enhanced by avoiding certain additive products that are known to reduce conductivity, or even adding e.g. polymer or metallic fibres to the concrete mix [49]. Hence, a range $\lambda_{c}=1.5-3 \mathrm{~W} /(\mathrm{m} \cdot \mathrm{K})$ was selected based on previous literature (e.g. [5]). All these pile design parameters are expected to exhibit a significant impact in their energy performance, as corroborated by previous research 
results for standard diameter rotary bored piles [5]. However, their relative importance may change for EMPs, due to the differences in typical sizes as well as reinforcement and U-loop arrangements.

Other design parameters that were considered concern the primary circuit of the GSHP system, namely the pipe diameter $d_{\text {pipe }}$, the fluid velocity $v$, the fluid thermal conductivity $\lambda_{f}$ and the thermal conductivity of the pipe material $\lambda_{p}$. Being virtually free from structural and geotechnical constraints, these parameter values may be relatively more freely selected by the geothermal designer, to optimize heat exchange. However, limitations still exist due to fluid circuit design criteria and the properties of involved materials. A range of 12-20 $\mathrm{mm}$ was chosen for $d_{\text {pipe }}$, reflecting the smaller overall diameter of EMPs compared to standard EPs, the lower and upper bound values corresponding to the diameter adopted in the EMP prototype described in [26], and the lower bound value used in standard EPs [50], respectively. Fluid velocity $v$ is easily controlled via circulation pump and may be considered the least constrained parameter, however in this work it was set to vary between 0.4 and $1.2 \mathrm{~m} / \mathrm{s}$, following Cecinato \& Loveridge [5], to allow for the development of turbulent flow (Reynolds number $R e>5500$ ) whilst not becoming unrealistically large. Parameter $\lambda_{p}$ clearly depends on the choice of pipe material, hence it can be considered to range between the conductivity of two common materials, namely standard HDPE and stainless steel, resulting in $\lambda_{p}=0.4-20 \mathrm{~W} /(\mathrm{m} \cdot \mathrm{K})$. The possible range of variability of $\lambda_{f}$ is rather narrow, as the typical heat carrier fluid can be either pure water or an antifreeze solution, depending on the expected operating temperatures (e.g. the use of anti-freeze is unavoidable in Northern European countries for winter operation, but may be avoided in Mediterranean areas). However, nanofluid based additives have been recently proposed (e.g. [51]-[53]) to enhance water's thermal conductivity in geothermal applications. Based on these examples, a range of $\lambda_{f}=0.6-0.79$ $\mathrm{W} /(\mathrm{m} \cdot \mathrm{K})$ was chosen.

On the other hand, parameters that cannot be changed at a given construction site (e.g., soil thermo-physical properties), were kept constant and equal to average values (Table 2).

Table 2. Fixed parameters adopted in the set \#1 parametric study simulations.

\begin{tabular}{l|c|c|c|c} 
Parameters & Thermal conductivity & Specific heat capacity & Density & Porosity \\
\hline Units & $\mathrm{W} /(\mathrm{m} \cdot \mathrm{K})$ & $\mathrm{J} /(\mathrm{kg} \cdot \mathrm{K})$ & $\mathrm{kg} / \mathrm{m}^{3}$ & -- \\
\hline Soil & 1.34 & 800 & 1835 & 0.3 \\
\hline Concrete & $($ See Table 1$)$ & 880 & 2300 & 0.0 \\
\hline Steel & 45 & 475 & 7850 & 0.0 \\
\hline
\end{tabular}

\subsubsection{Site dependent parameters}

Site dependent parameters were chosen as the ones that i) are expected to exert a significant influence on the energy performance of EMPs and ii) depend on the pre-existing situation at the construction site and cannot be changed by design arrangements. These factors are deemed of particular importance for micropile foundation solutions, which are often 
adopted for the retrofitting of existing buildings, hence neither the thermal properties of soil (even excavation of the surficial layers and subsequent backfill are generally not possible) nor those of the overlying structure can be engineered. The above outlined criteria led to the identification of a smaller set of parameters (summarized in Table 3) compared to design dependent ones, namely: ground thermal conductivity $\lambda_{g}$ and specific heat capacity at constant pressure $c_{p g}$, the air temperature boundary condition at ground level $T_{a}$ and the degree of thermal insulation at ground level, represented by the thickness of a possible expanded polystyrene insulating layer (cf. Section 2.2), $t_{i}$.

Ground conductivity is considered among site parameters because it is known to be of paramount importance in geothermal problems, as well as being relatively easy to estimate or measure both via insitu and laboratory methods. Among the former methods, Thermal Response Testing (TRT) is the best established for traditional borehole heat exchanger applications (e.g. see [31]), while its applicability to large diameter EPs (i.e., larger than 300-450 mm) has been questioned by several authors (e.g. see [54], [55]) due to cost barriers (the TRT duration is proportional to the necessary time to reach steady-state, in turn proportional to EP diameter) and the inherent simplifications in the analytical TRT interpretation methods (i.e. representing the EP as a linear heat source, despite its typically stocky aspect ratio). However EMPs, given their small diameter (not much larger than that of a BHE), can be deemed suitable to economically and reliably undergo TRTs (as is also shown in [26]). Laboratory methods including guarded hot plate, needle probe, comparative cut-bar tests, etc. are less reliable in assessing ground thermal conductivity, due to the well-known sample scale and homogeneity issues (e.g. see [31]). Geomaterials exhibit a wide range of variability of $\lambda_{g}$ depending on several factors like mineralogy, water content/saturation and porosity. Since in this work we are focusing on EPs installed in fine-grained soils, which incidentally are the most frequently involved in differential settlement/foundation underpinning problems, a typical thermal conductivity range for clays was selected after Banks [56], as $\lambda_{g}=0.9-2.2 \mathrm{~W} /(\mathrm{m} \cdot \mathrm{K})$.

Table 3. Set \#2 of site dependent parameters and relevant ranges of variation.

\begin{tabular}{l|c|c|c|c} 
Parameters & $\begin{array}{c}\text { Ground thermal con- } \\
\text { ductivity } \lambda_{g}\end{array}$ & $\begin{array}{c}\text { Ground specific heat } \\
\text { capacity } c_{p g}\end{array}$ & $\begin{array}{c}\text { Thickness of insulating layer } \\
t_{i}\end{array}$ & $\begin{array}{c}\text { Air temperature } \\
T_{a}\end{array}$ \\
\hline Units & $\mathrm{W} /(\mathrm{m} \cdot \mathrm{K})$ & $\mathrm{J} /(\mathrm{kg} \cdot \mathrm{K})$ & $\mathrm{mm}$ & ${ }^{\circ} \mathrm{C}$ \\
\hline Lower bound (MIN) & 0.9 & 1100 & 100 & 15 \\
\hline Middle bound (MED) & 1.6 & 1870 & 200 & 22 \\
\hline Upper bound (MAX) & 2.2 & 2640 & & 30 \\
\hline
\end{tabular}

The role of ground specific heat capacity $c_{p g}$ is not as established as that of $\lambda_{g}$, neither it is as frequently measured in geothermal practice. In fact, $c_{p g}$ is relevant to transient heat transfer analyses only. However, the typical wavy nature of temperature histories experienced by shallow geothermal installations suggests that $c_{p g}$ can play a potentially important role in the short- and medium-term energy performance of EMPs. Moreover, estimation/measurement methods for this soil parameter exist, either in the laboratory (e.g. [57], [58]) or by numerical back-analysis of TRT results (e.g. [59]). 
Alternatively, $c_{p g}$ may be calculated from the known porosity and heat capacities of water and the solid grains, as explained in Section 2.2. A reasonable range of variability obtained experimentally for clayey soils can be established following Clarke et al. [58] as $1100-2640 \mathrm{~J} /(\mathrm{kg} \cdot \mathrm{K})$.

Parameters $T_{a}$ and $t_{i}$ should be considered part of the (geo)thermal system design in any new construction project, i.e., they could be both engineered to some extent, based on the designated use of internal space and thermal insulation criteria. However, most typical micropile applications involve the retrofitting of existing buldings of different age, ranging from a few years old to historic (possibly even protected by Architectural Heritage bounds). To reflect such peculiar aspect of EMP utilization, $T_{a}$ and $t_{i}$ are considered in this work as site-dependent parameters.

The average air temperature at the top of the foundation may vary depending on whether the pre-existing foundation plane lies at the bottom of an excavation or is level with the surrounding ground, and on the use of internal space (e.g. car parking or cloister vs air-conditioned room). To allow for such variability, realistic lower and upper bounds for $T_{a}$ can be set as the minimum and maximum summertime average air temperature at the ground recorded in the Southern European zone (Rome), $T_{a}=15-30^{\circ} \mathrm{C}$ (e.g. see [44]).

Parameter $t_{i}$ is set to represent the degree of thermal insulation at the ground-air interface. While in practice a variety of different materials and flooring arrangements can occur, it seems sensible to assume $t_{i}=0$ as a lower bound, in the absence of any thermal insulation, and $t_{i}=200 \mathrm{~mm}$ as an upper bound, representing a high degree of thermal insulation following current practice in 'thermal coating' of buildings (e.g. see [43]).

\subsection{Design of parametric analysis}

Given the number of parameters involved in the two sets defined above, running a numerical simulation for each possible combination of factors would constitute a prohibitive task. Hence, to more efficiently explore parameter space, resort is made to the theory of Engineering Statistics, with particular reference to the so-called Experimental Design method, that deals with purposely changing one or more variables in a process, to observe the effect of such changes on a response variable. Among the existing Experimental Design methods, the so-called Taguchi method was chosen for its reliability and adaptability to engineering problems [5], [7], [60]-[62]. A first step in the design of a Taguchi analysis is the definition of a suitable 'orthogonal array', i.e., a 2-dimensional matrix defining the variable settings for each of the numerical simulations needed (e.g., in Table 4 andTable 5 the orthogonal arrays 'L8' and 'L9' are respectively shown). Each row of the matrix contains the list of settings for all parameters in one simulation. The set of values (or 'levels') that each parameter can assume must be also defined. Each column of the array corresponds to one parameter and contains all the levels that will be assigned to the parameter during the numerical simulations. The most important property of an orthogonal array is 'statistical independence': not only within each column there is an equal number of occurrences for each level, but also the columns are mutually orthogonal, i.e. for each level within one column, each level within any 
other column will occur an equal number of times. Thanks to statistical independence, the estimation of the effect of any individual parameter tends to be accurate and reproducible [61].

To analyse the two parameter sets outlined in Section 3.1 in the frame of the Taguchi method, the number of 'levels' for each parameter and the 'response variable' of the numerical simulations must be defined. As the latter should be representative of the EMP energetic performance, the total exchanged power $Q$ (corresponding to the total heat flux, integrated over the EMP surface) after a given duration of geothermal operation was chosen as a suitable output variable. To represent the short-, medium-, and long-term operation of the EMP, the response variable $Q$ was computed after 3, 60 and 180 days of heat injection (see Section 2.2), respectively. As regards the number of levels, two levels (namely, the lower and upper bounds) per parameter were chosen for parameter set \#1, while three levels (namely the lower and upper bounds, and a mid-range value) for each parameter were chosen for parameter set \#2. Including in the analysis mid-range values in addition to extreme ones is useful to evaluate any non-linearity that may arise over each parameter's range, while not bringing about a significant computational overload, given the small number of parameters involved in set \#2.

The above outlined settings imply a first parametric analysis consisting of seven parameters with two levels each, and a second analysis involving four parameters with three levels each. The corresponding 'L8' and 'L9' Taguchi orthogonal arrays are readily available in the literature (e.g. see [61]), as shown in Table 4 and Table 5. It can be observed that the parameters to be investigated correspond to the array's columns, while the settings for each of the simulations required are reported in the array's rows. For each parameter, the levels are referred to as MIN, MED (only for set \#2), and MAX. The arrays can thus be filled in with the parameters' settings from Table 1 and Table 3 to finalise the parametric study design, as shown in Table 6 and Table 7.

With the above settings, the Taguchi parametric analysis \#1 and \#2 will need respectively only eight and nine simulations to be completed, followed by some basic statistical analysis of the results (so-called level average analysis, see Section 3.3). In contrast, running a simulation for each one of the possible combinations of parameters, so-called full factorial design (e.g. [61]) would imply a total number of simulations of $2^{7}=128$ for set $\# 1$ and $3^{4}=81$ for set $\# 2$. The advantage of adopting the Taguchi statistical method is thus apparent, as it allows significant time saving while ensuring the significance of results. Moreover, the Taguchi method also allows to double-check the reliability of a parametric analysis by performing confirmation runs (see Section 3.3 and [5], [61], [62]).

Table 4. 'L8' Taguchi orthogonal array involving seven parameters with two levels each.

\begin{tabular}{c|c|c|c|c|c|c|c} 
Run \# & Parameter 1 & Parameter 2 & Parameter 3 & Parameter 4 & Parameter 5 & Parameter 6 & Parameter 7 \\
\hline 1 & MIN & MIN & MIN & MIN & MIN & MIN & MIN \\
\hline 2 & MIN & MIN & MIN & MAX & MAX & MAX & MAX \\
\hline 3 & MIN & MAX & MAX & MIN & MIN & MAX & MAX \\
\hline 4 & MIN & MAX & MAX & MAX & MAX & MIN & MIN \\
\hline
\end{tabular}




\begin{tabular}{l|l|l|l|l|l|l|l}
\hline 5 & MAX & MIN & MAX & MIN & MAX & MIN & MAX \\
\hline 6 & MAX & MIN & MAX & MAX & MIN & MAX & MIN \\
\hline 7 & MAX & MAX & MIN & MIN & MAX & MAX & MIN \\
\hline 8 & MAX & MAX & MIN & MAX & MIN & MIN & MAX \\
\hline
\end{tabular}

Table 5. 'L9' Taguchi orthogonal array involving four parameters with three levels each.

\begin{tabular}{c|c|c|c|c} 
Run \# & Parameter 1 & Parameter 2 & Parameter 3 & Parameter 4 \\
\hline 1 & MIN & MIN & MIN & MIN \\
\hline 2 & MIN & MED & MED & MED \\
\hline 3 & MIN & MAX & MAX & MIN \\
\hline 4 & MED & MIN & MED & MAX \\
\hline 5 & MED & MED & MAX & MIN \\
\hline 6 & MED & MAX & MIN & MED \\
\hline 7 & MAX & MIN & MAX & MED \\
\hline 8 & MAX & MED & MIN & MAX \\
\hline 9 & MAX & MAX & MED & MIN \\
\hline
\end{tabular}

\subsection{Results and discussion}

The results of the eight runs for set \#1 and nine runs for set \#2 in terms of exchanged power Q vs time are shown in Figure 3 and Figure 4 for set \#1 and set \#2, respectively, and are also numerically reported after 1, 60 and 180 days of continuous heat injection in Table 6 and Table 7. These rather diverse values constitute the 'raw output data' of the parametric study, to which statistical post-processing needs to be applied in order to extract significant results. This is done via the so-called level average analysis [61], consisting of (i) calculating the average simulation result for each level of each parameter, (ii) quantifying the effect of each parameter by taking the absolute difference between the highest and lowest average results and (iii) identifying the strong effects, by ranking the parameters from the largest to the smallest absolute difference. Results are summarised in the 'response tables', shown in Table 8 and Table 9 for set \#1 and set \#2 respectively.

Table 6. 'L8' array describing the parameter levels in each column and the simulation settings in each row, including the confirmation run, and the analysis outcomes in terms of exchanged power after 1, 60 and 180 days in the last three columns, for set \#1.

\begin{tabular}{c|c|c|c|c|c|c|c|c|c|c} 
& $\begin{array}{c}v \\
(\mathrm{~m} / \mathrm{s})\end{array}$ & $\begin{array}{c}\lambda_{p} \\
\mathrm{~W} /(\mathrm{m} \cdot \mathrm{K})\end{array}$ & $\begin{array}{c}\lambda_{f} \\
\mathrm{~W} /(\mathrm{m} \cdot \mathrm{K})\end{array}$ & $\begin{array}{c}D_{\text {pile }} \\
\mathrm{mm}\end{array}$ & $\begin{array}{c}L \\
\mathrm{~m}\end{array}$ & $\begin{array}{c}\lambda_{C} \\
\mathrm{~W} /(\mathrm{m} \cdot \mathrm{K})\end{array}$ & $\begin{array}{c}d_{\text {pipe }} \\
\mathrm{mm}\end{array}$ & $\begin{array}{c}Q_{(1 d)} \\
\mathrm{W}\end{array}$ & $\begin{array}{c}Q_{(60 d)} \\
\mathrm{W}\end{array}$ & $\begin{array}{c}Q_{(180 d)} \\
\mathrm{W}\end{array}$ \\
\hline Run_1 & 0.4 & 0.6 & 0.60 & 150 & 10 & 1.5 & 12 & 402.94 & 276.88 & 289.64 \\
\hline Run_2 & 0.4 & 0.6 & 0.60 & 200 & 20 & 3.0 & 20 & 1027.53 & 605.84 & 657.39 \\
\hline Run_3 & 0.4 & 20.0 & 0.79 & 150 & 10 & 3.0 & 20 & 573.06 & 353.23 & 372.89 \\
\hline Run_4 & 0.4 & 20.0 & 0.79 & 200 & 20 & 1.5 & 12 & 839.64 & 536.97 & 577.42 \\
\hline Run_5 & 1.2 & 0.6 & 0.79 & 150 & 20 & 1.5 & 20 & 968.53 & 577.36 & 623.51 \\
\hline Run_6 & 1.2 & 0.6 & 0.79 & 200 & 10 & 3.0 & 12 & 524.32 & 334.76 & 351.77 \\
\hline Run_7 & 1.2 & 20.0 & 0.60 & 150 & 20 & 3.0 & 12 & 980.85 & 580.37 & 628.09 \\
\hline Run_8 & 1.2 & 20.0 & 0.60 & 200 & 10 & 1.5 & 20 & 502.63 & 323.04 & 338.38 \\
\hline Confirmation & 1.2 & 0.6 & 0.79 & 200 & 20 & 3.0 & 20 & 1135.53 & 699.94 & 643.41 \\
\hline
\end{tabular}


Table 7. 'L9' array describing the parameter levels in each column and the simulation settings in each row, including the confirmation run, and the analysis outcomes in terms of exchanged power after 1, 60 and 180 days in the last three columns, for set $\# 2$.

\begin{tabular}{c|c|c|c|c|c|c|c} 
& $\begin{array}{c}\lambda_{g} \\
\mathrm{~W} /(\mathrm{m} \cdot \mathrm{K})\end{array}$ & $\begin{array}{c}c_{p g} \\
\mathrm{~J} /(\mathrm{kg} \cdot \mathrm{K})\end{array}$ & $\begin{array}{c}t_{i} \\
\mathrm{~mm}\end{array}$ & $\begin{array}{c}T_{a} \\
{ }^{\circ} \mathrm{C}\end{array}$ & $\begin{array}{c}Q_{(1 d)} \\
\mathrm{W}\end{array}$ & $\begin{array}{c}Q_{(60 d)} \\
\mathrm{W}\end{array}$ & $\begin{array}{c}Q_{(180 d)} \\
\mathrm{W}\end{array}$ \\
\hline Run_1 & 0.9 & 1100 & 0 & 15 & 813.26 & 455.02 & 405.82 \\
\hline Run_2 & 0.9 & 1870 & 100 & 23 & 868.24 & 462.5 & 403.54 \\
\hline Run_3 & 0.9 & 2640 & 200 & 30 & 921.06 & 486.16 & 430.90 \\
\hline Run_4 & 1.6 & 1100 & 100 & 30 & 1021.46 & 627.86 & 557.66 \\
\hline Run_5 & 1.6 & 1870 & 200 & 15 & 1089.84 & 655.18 & 584.90 \\
\hline Run_6 & 1.6 & 2640 & 0 & 23 & 1136.68 & 695.66 & 631.56 \\
\hline Run_7 & 2.2 & 1100 & 200 & 23 & 1154.96 & 756.14 & 683.62 \\
\hline Run_8 & 2.2 & 1870 & 0 & 30 & 1262.62 & 898.10 & 809.18 \\
\hline Run_9 & 2.2 & 2640 & 100 & 15 & 1263.48 & 822.42 & 731.34 \\
\hline Confirmation & 2.2 & 2640 & 0 & 30 & 1311.67 & 952.21 & 839.14 \\
\hline
\end{tabular}

The response table yields a ranking of parameters from the most to the least influent one in maximising the energy output (represented by variable $Q$ ) of EMPs. Due to the statistical nature of this type of analyses, the influence of the bottomranked parameters cannot be assessed with confidence [61], hence most attention should be given to the top half of parameters in the ranking.

Moreover, to validate the statistical approach adopted, a confirmation run (e.g. see [61]) was performed for both parametric studies, consisting of running a simulation adopting the most influential parameter settings, and checking that the outcome of the confirmation run $Q_{\text {conf }}$ was larger than any of the other outcomes (i.e. runs 1-8 for set \#1 and 1-9 for set \#2). The validity of the adopted approach, recalling its inherent statistical nature, is apparent by observing, in Figure 3 and Figure 4, that the exchanged power in the confirmation simulation is larger than the other outputs practically at all times (i.e. maximizing the thermal performance for the selected EMP operation periods of 1, 60 and $180 \mathrm{~d}$ ).

To further illustrate the characteristics of heat diffusion in the ground, example temperature contour lines in the domain's transversal cross-section extracted from set \#2 simulations are shown in Figure 5, for two different simulation times (5 and 180 days). The EMP structure can be identified in the central area, and the pipes' position can be located by the dotshaped temperature contours corresponding to the largest temperature. By comparing results for run 1 (Figure 5a-b) and run 8 (Figure $5 \mathrm{c}-\mathrm{d}$ ), it can be observed that a larger energy output (as brought about by run 8 compared to run 1 , see Figure 4) corresponds to a larger overall temperature within the EMP area, especially in the long term. Moreover, larger radial temperature gradients are observed for run 1 compared to run 8 , consistent with the significantly larger thermal conductivity of the latter. 


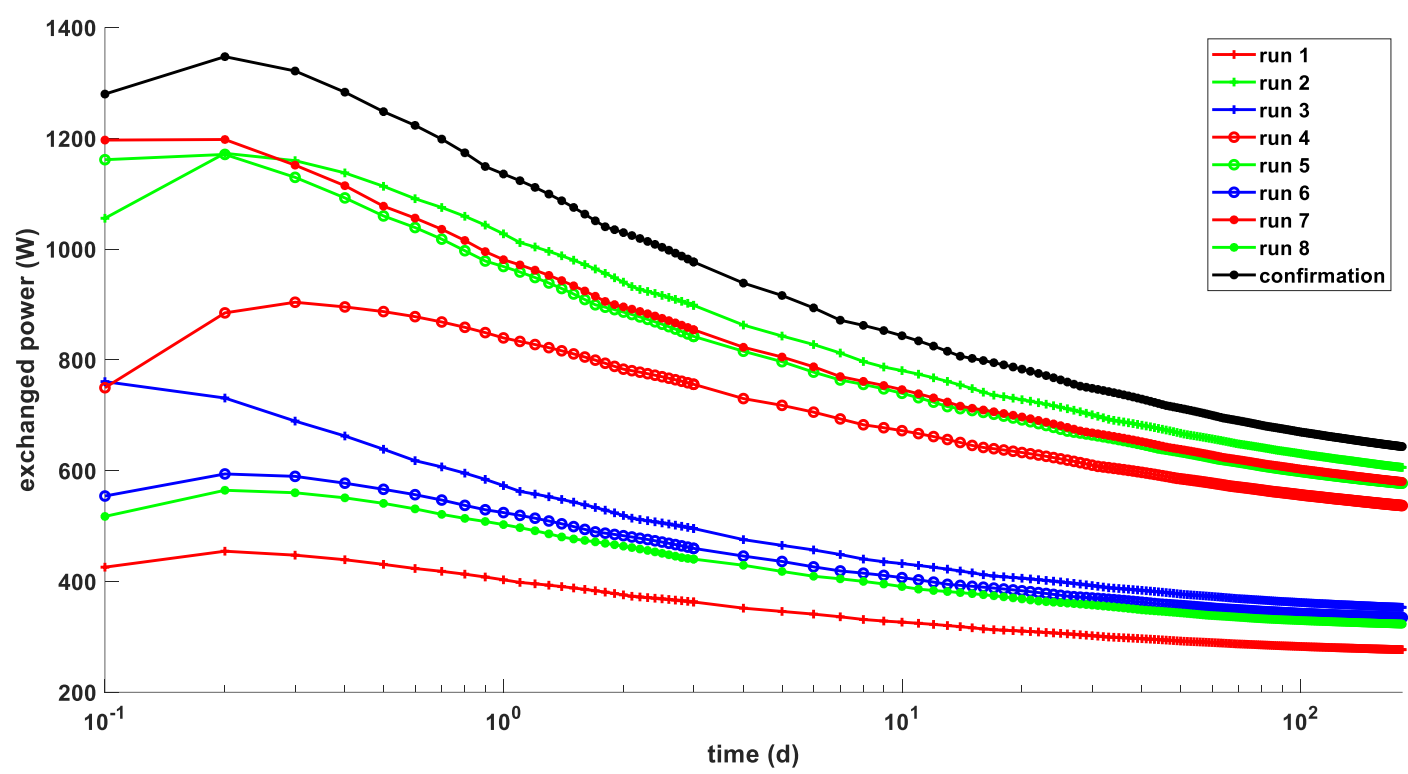

Figure 3. Simulated exchanged power vs time for runs 1-8 and confirmation run of the set \#1 parametric study.

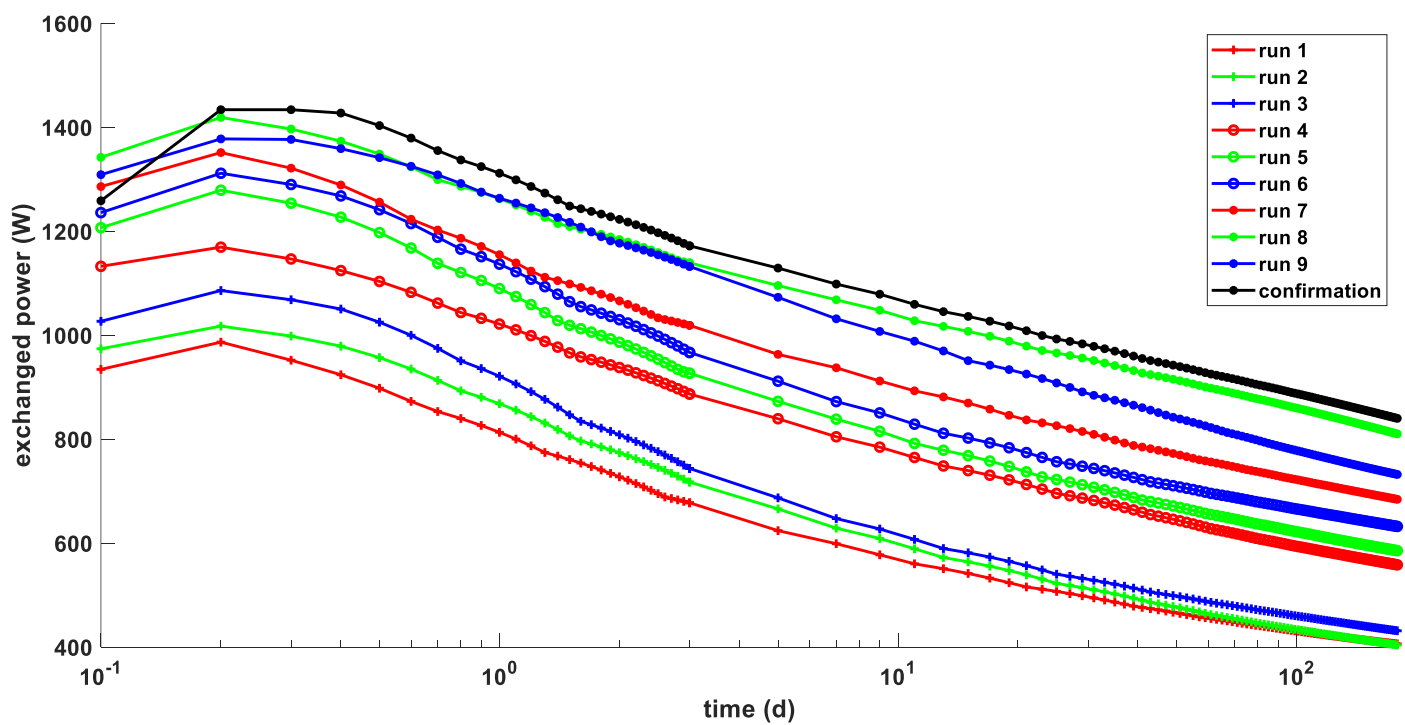

Figure 4. Simulated exchanged power vs time for runs 1-9 and confirmation run of the set \#2 parametric study. 

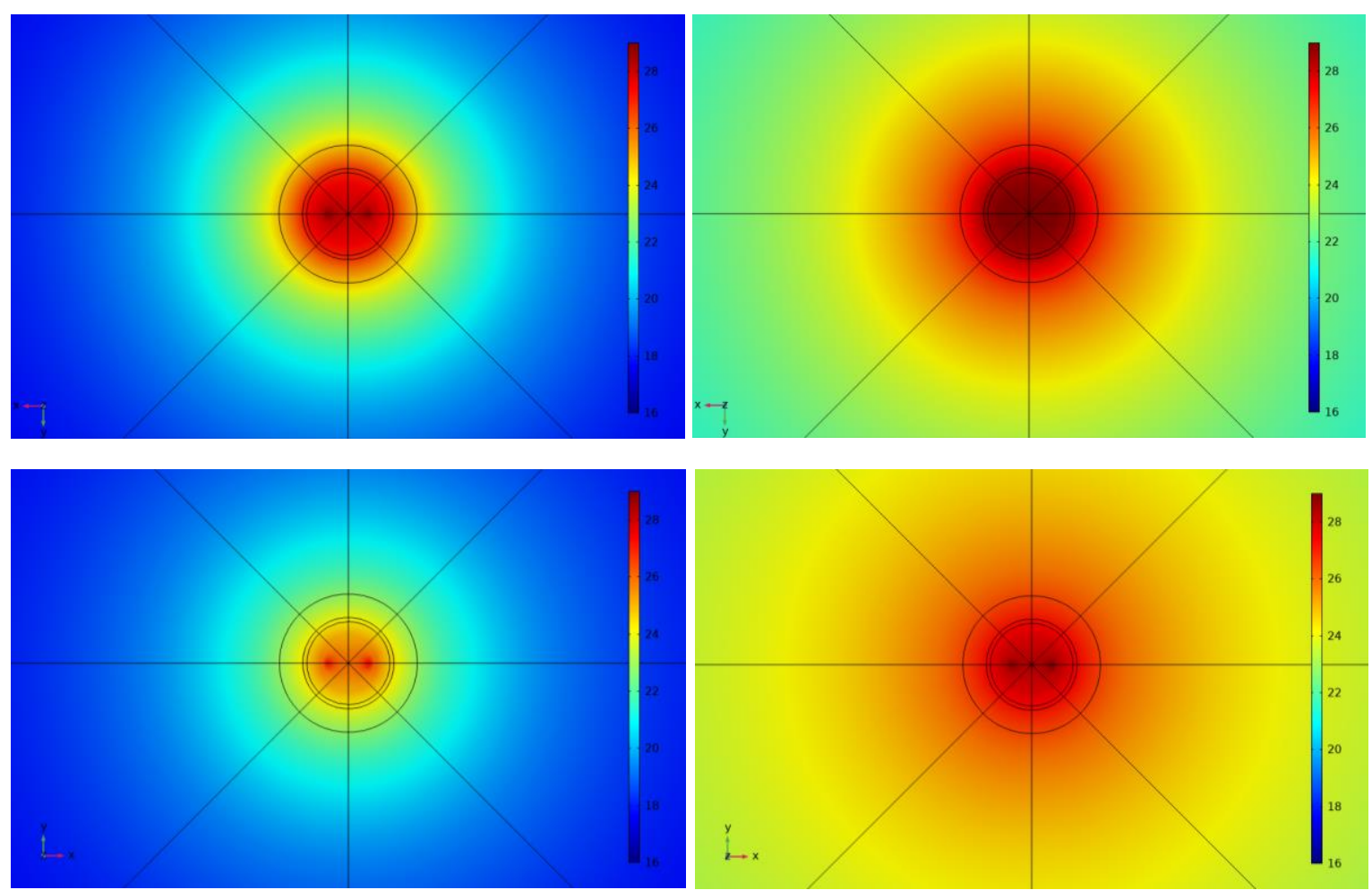

Figure 5. Example temperature contours (units: ${ }^{\circ} \mathrm{C}$ ) extracted from set \#2 simulations in the domain's transversal cross-section (EMP area), taken at $5 \mathrm{~m}$ below ground level, for run 1 after 5 days (a) and after 180 days (b), and for run 8 after 5 days (c) and after 180 days $(d)$.

Table 8. Response tables reporting level average analysis results in terms of exchanged power after 1, 60 and 180 days of heat injection, representing short-, medium- and long-term energy performance, for set \#1.

\begin{tabular}{c|c|c|c|c|c|c|c} 
& \multicolumn{8}{c}{ Short term: Level Average Analysis results after 1 day } \\
\cline { 2 - 9 } & $\begin{array}{c}v \\
(\mathrm{~m} / \mathrm{s})\end{array}$ & $\begin{array}{c}\lambda_{p} \\
\mathrm{~W} /(\mathrm{m} \cdot \mathrm{K})\end{array}$ & $\begin{array}{c}\lambda_{f} \\
\mathrm{~W} /(\mathrm{m} \cdot \mathrm{K})\end{array}$ & $\begin{array}{c}D_{\text {pile }} \\
\mathrm{mm}\end{array}$ & $\begin{array}{c}L \\
\mathrm{~m}\end{array}$ & $\begin{array}{c}\lambda_{C} \\
\mathrm{~W} /(\mathrm{m} \cdot \mathrm{K})\end{array}$ & $\begin{array}{c}d_{\text {pipe }} \\
\mathrm{mm}\end{array}$ \\
\hline Min & 710.79 & 730.83 & 728.49 & 731.34 & 500.74 & 678.44 & 686.94 \\
\hline Max & 744.08 & 724.05 & 726.39 & 723.53 & 954.14 & 776.44 & 767.94 \\
\hline Effect & 33.29 & 6.79 & 2.10 & 7.81 & 453.40 & 98.00 & 81.00 \\
\hline Ranking & 4 & 6 & 7 & 5 & 1 & 2 & 3 \\
\hline
\end{tabular}

\begin{tabular}{c|c|c|c|c|c|c|c|} 
& \multicolumn{8}{|c}{ Medium term: Level Average Analysis results after 60 days } \\
\cline { 2 - 9 } & $\begin{array}{c}v \\
(\mathrm{~m} / \mathrm{s})\end{array}$ & $\begin{array}{c}\lambda_{p} \\
\mathrm{~W} /(\mathrm{m} \cdot \mathrm{K})\end{array}$ & $\begin{array}{c}\lambda_{f} \\
\mathrm{~W} /(\mathrm{m} \cdot \mathrm{K})\end{array}$ & $\begin{array}{c}D_{\text {pile }} \\
\mathrm{mm}\end{array}$ & $\begin{array}{c}L \\
\mathrm{~m}\end{array}$ & $\begin{array}{c}\lambda_{C} \\
\mathrm{~W} /(\mathrm{m} \cdot \mathrm{K})\end{array}$ & $\begin{array}{c}d_{p i p e} \\
\mathrm{~mm}\end{array}$ \\
\hline Min & 474.33 & 480.57 & 478.37 & 478.53 & 338.17 & 457.24 & 461.73 \\
\hline Max & 485.44 & 479.20 & 481.40 & 481.24 & 621.60 & 502.53 & 498.04 \\
\hline Effect & 11.10 & 1.38 & 3.02 & 2.71 & 283.43 & 45.29 & 36.31 \\
\hline Ranking & 4 & 7 & 5 & 6 & 1 & 2 & 3 \\
\hline
\end{tabular}

\begin{tabular}{c|c|c|c|c|c|c|c|} 
& \multicolumn{8}{|c|}{ Long term: Level Average Analysis results after 180 days } \\
\cline { 2 - 9 } & $\begin{array}{c}v \\
(\mathrm{~m} / \mathrm{s})\end{array}$ & $\begin{array}{c}\lambda_{p} \\
\mathrm{~W} /(\mathrm{m} \cdot \mathrm{K})\end{array}$ & $\begin{array}{c}\lambda_{f} \\
\mathrm{~W} /(\mathrm{m} \cdot \mathrm{K})\end{array}$ & $\begin{array}{c}D_{\text {pile }} \\
\mathrm{mm}\end{array}$ & $\begin{array}{c}L \\
\mathrm{~m}\end{array}$ & $\begin{array}{c}\lambda_{C} \\
\mathrm{~W} /(\mathrm{m} \cdot \mathrm{K})\end{array}$ & $\begin{array}{c}d_{\text {pipe }} \\
\mathrm{mm}\end{array}$ \\
\hline Min & 443.23 & 448.71 & 446.53 & 446.96 & 321.98 & 428.56 & 432.24 \\
\hline Max & 453.88 & 448.40 & 450.58 & 450.15 & 575.13 & 468.55 & 464.87 \\
\hline Effect & 10.66 & 0.30 & 4.05 & 3.19 & 253.16 & 39.99 & 36.62 \\
\hline Ranking & 4 & 7 & 5 & 6 & 1 & 2 & 3 \\
\hline
\end{tabular}




\subsubsection{Impact of design parameters}

It is interesting to note that the three most important design factors in EMP energy performance are pile length, concrete conductivity and pipe diameter. The importance of pile length is very high, as expected, and confirmed by previous studies on standard EPs (e.g. see [5]). The second ranked factor is concrete conductivity, representing, as it also emerged in previous literature on EPs, the importance of concrete 'thermal resistance' in heat conduction. However, probably the most remarkable outcome of this analysis is the major importance of pipe diameter in maximising the energy performance of EMPs. This is in contrast with literature results on medium- and large-diameter bored piles [5] and can be explained by the fact that pipes occupy a larger portion of surface area within a micropile's cross-section compared to that of a standard pile. This result constitutes an important outcome to aid thermal design of EMPs. In fact, it is easier to decide the diameter of pipes independently of structural/geotechnical design, compared to the pile length (which is rarely changed just to provide additional energy supply) or concrete conductivity (which could be enhanced by selecting the aggregate lithology, although this is not common practice).

Table 9. Response tables reporting level average analysis results in terms of exchanged power after 1, 60 and 180 days of heat injection, representing short-, medium- and long-term energy performance, for set \#2.

\begin{tabular}{c|c|c|c|c} 
& \multicolumn{4}{|c}{ Short term: Level Average Analysis results after 1 day } \\
\cline { 2 - 5 } & $\begin{array}{c}\lambda_{g} \\
\mathrm{~W} /(\mathrm{m} \cdot \mathrm{K})\end{array}$ & $\begin{array}{c}c_{p g} \\
\mathrm{~J} /(\mathrm{kg} \cdot \mathrm{K})\end{array}$ & $\begin{array}{c}t_{i} \\
\mathrm{~mm}\end{array}$ & $\begin{array}{c}T_{a} \\
{ }^{\circ} \mathrm{C}\end{array}$ \\
\hline Min & 867.52 & 996.56 & 1070.85 & 1055.53 \\
\hline Med & 1082.66 & 1073.57 & 1051.06 & 1053.29 \\
\hline Max & 1227.02 & 1107.07 & 1055.287 & 1068.38 \\
\hline Effect & 359.50 & 110.51 & 15.57 & 15.09 \\
\hline Ranking & 1 & 2 & 3 & 4 \\
\hline
\end{tabular}

\begin{tabular}{c|c|c|c|c}
\multirow{2}{*}{} & \multicolumn{4}{|c}{ Medium term: Level Average Analysis results after 60 days } \\
\cline { 2 - 5 } & $\begin{array}{c}\lambda_{g} \\
\mathrm{~W} /(\mathrm{m} \cdot \mathrm{K})\end{array}$ & $\begin{array}{c}c_{p g} \\
\mathrm{~J} /(\mathrm{kg} \cdot \mathrm{K})\end{array}$ & $\begin{array}{c}t_{i} \\
\mathrm{~mm}\end{array}$ & $\begin{array}{c}T_{a} \\
{ }^{\circ} \mathrm{C}\end{array}$ \\
\hline Min & 467.89 & 613.01 & 682.93 & 644.21 \\
\hline Med & 659.57 & 671.93 & 637.59 & 638.10 \\
\hline Max & 825.55 & 668.08 & 632.49 & 670.71 \\
\hline Effect & 357.66 & 58.92 & 50.43 & 32.61 \\
\hline Ranking & 1 & 2 & 3 & 4 \\
\hline
\end{tabular}

\begin{tabular}{c|c|c|c|c}
\multirow{2}{*}{} & \multicolumn{4}{|c}{ Long term: Level Average Analysis results after 180 days } \\
\cline { 2 - 5 } & $\begin{array}{c}\lambda_{g} \\
\mathrm{~W} /(\mathrm{m} \cdot \mathrm{K})\end{array}$ & $\begin{array}{c}c_{p g} \\
\mathrm{~J} /(\mathrm{kg} \cdot \mathrm{K})\end{array}$ & $\begin{array}{c}t_{i} \\
\mathrm{~mm}\end{array}$ & $\begin{array}{c}T_{a} \\
{ }^{\circ} \mathrm{C}\end{array}$ \\
\hline Min & 413.42 & 549.03 & 615.52 & 574.02 \\
\hline Med & 591.37 & 599.21 & 564.18 & 572.91 \\
\hline Max & 741.38 & 597.93 & 566.47 & 599.25 \\
\hline Effect & 327.96 & 50.17 & 51.34 & 26.34 \\
\hline Ranking & 1 & 3 & 2 & 4 \\
\hline
\end{tabular}


The above outcomes corroborate the importance of carrying out bespoke parametric analyses to assess the influences on the energy performance of EMPs. While it has been shown that the overall energy performances of EMPs and standard EPs are comparable in terms of heat exchange rate per unit length [26], it is useful to assess the relative importance of different design parameter settings to optimize heat exchange. In this respect, different geothermal design criteria should be adopted for EMPs and EPs. For the latter, maximizing the number of U-pipes (ranked 1st out of 7 in the Taguchi analysis of Cecinato \& Loveridge [5]) is recommended, consistent with maximising the pile diameter (ranked 4th out of 7 in the Taguchi analysis of Cecinato \& Loveridge [5]). For EMPs, neither of these settings would be effective, since varying their diameter within the small available range does not impact thermal results, and adopting more than a single U-pipe would be detrimental for i) space constraints within their cross-section (also possibly affecting their structural performance) and ii) for the likely occurrence of thermal interference among a number of pipes that are too close together [5], [6]. On the other hand, the pipe diameter in EPs has negligible impact (ranked 6th out of 7 in the Taguchi analysis of [5]), while it has a large impact in EMPs (ranked 3rd in Table 8), as well as being the single easiest parameter to engineer based on geothermal only criteria.

It should be remarked that the above discussed comparison between results of set \#1 parametric study for EMPs, and those for EPs discussed in a previous publication [5], is based on the fact that the same numerical (and statistical) approach was used in both studies. Namely, in both studies a FEM numerical model (describing transient heat conduction in the solids and convective heat exchange between the heat-carrier fluid and pipe walls) was employed to carry out a Taguchi parametric study, to identify the dominant design parameters in maximizing the thermal performance of the specific type of energy geostructure.

Moreover, fluid's velocity and thermal conductivity come fourth and fifth in the ranking respectively, so they can be considered to exert a mild, yet tangible, influence on the thermal performance of EMPs. The latter may be enhanced by using particular additives such as nanofluids (e.g. [52]), while the former is controlled by circulation pump settings. However, it has been shown [5] that increasing the heat transfer fluid velocity does not have a major impact on the overall exchanged energy, providing turbulent flow is maintained within the pipes. In addition, it should be borne in mind that increasing fluid velocity beyond reasonable limits alters the global energy balance, since it increases the circulating pump's energy expenditure.

Regarding the reference time frame of geothermal operation, no appreciable changes are observed between short and medium-long term operation. In fact, Table 8 shows that the top half of parameters in the level average rankings (i.e., the 
ones with a significant impact in the results) remain unchanged after 1, 60 and 180 days. Hence, the bulk of the above discussed results holds both in transient and in (quasi) steady-state conditions.

It is worth remarking that the above discussed top-5 dominant design parameters maximise EMP energy output when they are set to their largest values. Hence in general, for an energy efficient EMP thermal design, it should be made sure that EMP's length, pipe diameter, concrete conductivity, and fluid conductivity and velocity are maximised.

\subsubsection{Impact of site parameters}

The outcome of the set\#2 parametric study illustrated by the corresponding level average analysis (Table 9) confirms, first of all, the dominant role of soil thermal conductivity in maximising EMP energy performance both for short- and long-term operation. This result is in agreement with the bulk of shallow geothermal energy literature, as well as with most studies specifically concerning energy geostructures (e.g. [59]). Hence, whenever a given site is to be assessed for its suitability for an EMP solution, the conductivity of the ground affected by potential heat exchange should be carefully measured or estimated.

On the other hand, Table 9 shows that the air temperature exhibits the least relative impact in the exchanged power, since it ranks consistently in the last position at all considered time frames. This outcome may be explained with the fact that air temperature affects a boundary of the domain that is not in direct contact with the EMP outer surface, while the soil mass acts as a buffer to heat exchange between the heat source and the ground level boundary. This suggests that an EMP solution can be conveniently adopted regardless of the type of (indoor or outdoor) space above the foundation plane. As an example, the energy efficiency of the geothermal solution will be practically the same if the micropiles are to be installed within a historic arcade or in the air-conditioned basement of a modern building.

However, level average analyses provide more interesting insights when considering site parameters that come second and third in the rankings. In particular, in the short-term analysis, soil specific heat capacity is ranked second, while it is ranked third in the long term. This result is consistent with the fact that $c_{p g}$ is relevant to the transient heat transfer phase, while it becomes irrelevant at steady state. As a result, for a given site, measuring or estimating ground's $c_{p g}$ is advisable, as larger specific heat values will enhance the EMP efficiency during transient operation. However, this parameter should be considered of secondary importance, since i) it is more difficult (and less usual) to measure compared to $\lambda_{g}$, and ii) the relatively small diameter of EMPs mean that they can quickly reach steady state compared to standard EPs (e.g. [54]).

The insulation layer thickness, representing the degree of existing thermal insulation between the ground and the building, is ranked third in the short term, but becomes the second most important parameter in the long term. This is a peculiar 
result, not only because $t_{i}$ is usually not considered among relevant factors governing thermal efficiency of energy geostructures but also because, counter-intuitively, better thermal performance is brought about by a lesser extent (or absence) of insulation. This is illustrated in Figure 6, and could be explained by the fact that the insulating layer inhibits heat exchange between the ground and the atmosphere, thus in the long term the temperature of the upper part of the soil-pile domain tends to build up at a much higher rate compared to the rest of the domain. This, in turn, reduces the long-term efficiency of heat injection. It can be deduced that, contrary to common engineering intuition, the presence of an insulating layer between the ground and the overlying building space decreases the thermal efficiency of EMPs (although it might improve the overall efficiency of the space heating/cooling system, based on thermal system engineering criteria that are outside the scope of this work). This suggests that typical site conditions where micropile solutions are required, i.e. in the context of retrofitting of old buildings lacking modern 'thermal coating' (exterior insulation) solutions, are favourable for the installation of energy-efficient EMP solutions.

In summary, the short-term energy performance of EMPs is maximized when EMPs are installed in a soil characterized by large thermal conductivity and specific heat capacity, as intuitively expected, while the other site parameter values do not exert a significant influence. In the long-term, however, in addition to a highly conductive ground, it is the absence (or scarcity) of thermal insulation between the ground and the overlying space that enhances EMP thermal output the most. 

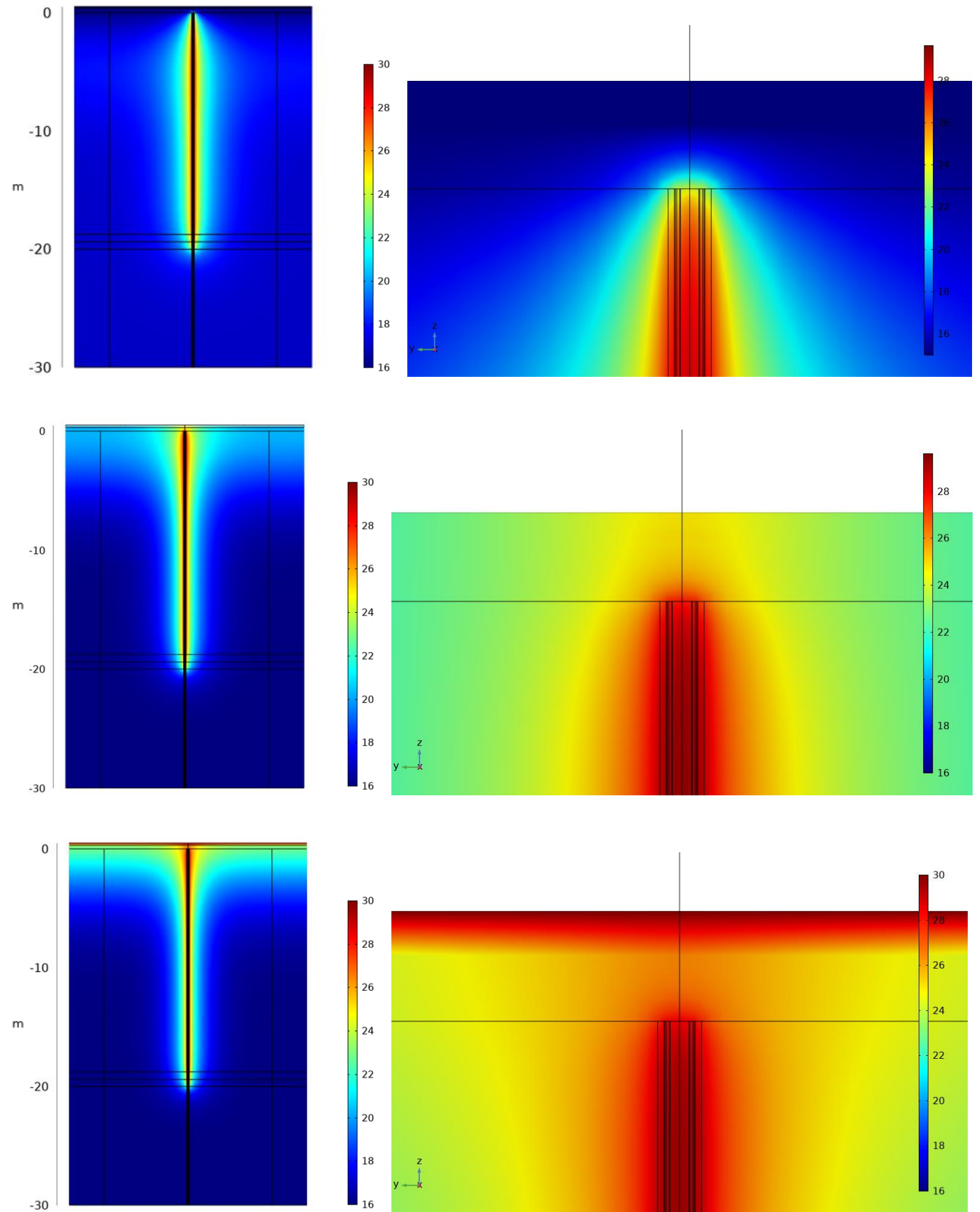

Figure 6. Temperature contour lines after $180 \mathrm{~d}$ of heat injection for set \#2 (a) run 1 (no insulation layer), (b) run 2 ( $t_{i}=10 \mathrm{~cm}$ ), (c) run $3\left(t_{i}=20 \mathrm{~cm}\right)$. While images on the left show a longitudinal $2 D$ section of the whole integration domain, those on the right show close-up views of the pile head and central slab area, to further appreciate the effect of boundary conditions in the heat diffusion process. 


\section{Conclusions}

In this work, a numerical model was employed in conjunction with statistical analysis to perform a systematic study considering the parameters governing the energy performance of thermo-active micro-piles, representing a promising emerging technology, able to combine structural and energy retrofitting of buildings. Reference was made to a known case study, presented by Ronchi et al. [26]. The following conclusive observations can be made.

- As for the design dependent parameters (set \#1), a major role is played (both for short- and long-term operation), in order of importance, by the pile length, concrete conductivity and pipe diameter. While the first two aspects are also relevant to the design of standard EPs, the third one is an element that assumes greater importance for EMPs, due to the different ratio between the diameter of the pile and that of the pipes. In general, results show that thermal design of EMPs should not be based on the same criteria as those used for EP design, since different parameters are dominant in enhancing their energy performance. In particular, the pipe diameter emerged as a key factor, and should be maximized in EMPs for its strong influence in results. This design feature is rather convenient to apply in practice, especially as long as it is independent of structural/geotechnical criteria.

- As regards the site dependent parameters (set \#2), the dominant role of ground thermal conductivity in maximising the energy performance of EMPs is confirmed, as it has been observed by numerous authors for other types of EGS. Hence, a careful experimental measurement or estimation of the conductivity of involved soil(s) is recommended for a reliable assessment of the performance of an EMP system. Moreover, somewhat counter to engineering intuition, when long-term EMP operation is considered, the extent of thermal insulation between the ground and the building becomes the second most important parameter in EMP energy performance, the absence of insulation emerging as the most favourable situation. Hence, EMPs can be conveniently used in energy (and structural) retrofitting of old buildings even though they are not equipped with an exterior basement insulation system.

On the other hand, challenges still remain concerning the installation of EMPs, mainly due to the on-site constraints in existing buildings, and this is one of the topics of future research. Every underpinning project to be implemented with EMPs should be studied individually, and beside technical feasibility, the economical one must obviously be considered. In any case, EMPs can be generally considered a viable and convenient geothermal solution, as with other EGS types, since they remove the cost of ad-hoc excavations while being in any case required for structural/geotechnical reasons.

Acknowledgements Ms Melissa Monni is gratefully acknowledged for carrying out the set \#1 numerical simulations. 


\section{References}

[1] H. Brandl, "Energy foundations and other thermo-active ground structures," Geotechnique, vol. 56, no. 2, pp. 81122, 2006, doi: 10.1680/geot.2006.56.2.81.

[2] N. Batini, A. F. Rotta Loria, P. Conti, D. Testi, W. Grassi, and L. Laloui, "Energy and geotechnical behaviour of energy piles for different design solutions," Appl. Therm. Eng., vol. 86, pp. 199-213, 2015, doi: 10.1016/j.applthermaleng.2015.04.050.

[3] F. Dupray, L. Laloui, and A. Kazangba, "Numerical analysis of seasonal heat storage in an energy pile foundation," Comput. Geotech., vol. 55, pp. 67-77, 2014, doi: 10.1016/j.compgeo.2013.08.004.

[4] P. J. Bourne-Webb, B. Amatya, K. Soga, T. Amis, C. Davidson, and P. Payne, "Energy pile test at lambeth college, London: Geotechnical and thermodynamic aspects of pile response to heat cycles," Geotechnique, vol. 59, no. 3, pp. 237-248, 2009, doi: 10.1680/geot.2009.59.3.237.

[5] F. Cecinato and F. A. Loveridge, "Influences on the thermal efficiency of energy piles," Energy, vol. 82, 2015, doi: 10.1016/j.energy.2015.02.001.

[6] F. Loveridge and F. Cecinato, "Thermal performance of thermoactive continuous flight auger piles," Environ. Geotech., vol. 3, no. 4, 2016, doi: 10.1680/jenge.15.00023.

[7] A. Di Donna, F. Cecinato, F. Loveridge, and M. Barla, "Energy performance of diaphragm walls used as heat exchangers," Proc. Inst. Civ. Eng. Geotech. Eng., vol. 170, no. 3, 2017, doi: 10.1680/jgeen.16.00092.

[8] D. Salciarini, F. Ronchi, E. Cattoni, and C. Tamagnini, "Thermomechanical Effects Induced by Energy Piles Operation in a Small Piled Raft," Int. J. Geomech., vol. 15, no. 2, p. 04014042, Apr. 2015, doi: 10.1061/(asce)gm.1943-5622.0000375.

[9] D. Salciarini, F. Ronchi, and C. Tamagnini, "Thermo-hydro-mechanical response of a large piled raft equipped with energy piles: a parametric study," Acta Geotech., vol. 12, no. 4, pp. 703-728, 2017, doi: 10.1007/s11440017-0551-3.

[10] R. Caulk, E. Ghazanfari, and J. S. McCartney, "Parameterization of a calibrated geothermal energy pile model," Geomech. Energy Environ., vol. 5, pp. 1-15, 2016, doi: 10.1016/j.gete.2015.11.001.

[11] O. Ogunleye, R. M. Singh, F. Cecinato, and J. Chan Choi, "Effect of intermittent operation on the thermal efficiency of energy tunnels under varying tunnel air temperature," Renew. Energy, vol. 146, 2020, doi: 10.1016/j.renene.2019.08.088.

[12] A. Insana and M. Barla, "Experimental and numerical investigations on the energy performance of a thermoactive tunnel," Renew. Energy, vol. 152, pp. 781-792, 2020, doi: https://doi.org/10.1016/j.renene.2020.01.086.

[13] M. Barla and A. Di Donna, "Energy tunnels: concept and design aspects," Undergr. Sp., vol. 3, no. 4, pp. 268276, 2018, doi: https://doi.org/10.1016/j.undsp.2018.03.003.

[14] A. Di Donna and M. Barla, "The role of ground conditions on energy tunnels' heat exchange," Environ. Geotech., vol. 3, no. 4, pp. 214-224, 2016, doi: 10.1680/jenge.15.00030.

[15] N. Makasis, G. A. Narsilio, A. Bidarmaghz, I. W. Johnston, and Y. Zhong, "The importance of boundary conditions on the modelling of energy retaining walls," Comput. Geotech., vol. 120, p. 103399, 2020.

[16] D. Sterpi, G. Tomaselli, and A. Angelotti, "Energy performance of ground heat exchangers embedded in diaphragm walls: Field observations and optimization by numerical modelling," Renew. Energy, vol. 147, no. xxxx, pp. 2748-2760, 2020, doi: 10.1016/j.renene.2018.11.102.

[17] S. Dong et al., "Thermo-mechanical behavior of energy diaphragm wall: Physical and numerical modelling," Appl. Therm. Eng., vol. 146, pp. 243-251, 2019.

[18] A. F. Rotta Loria and L. Laloui, "Group action effects caused by various operating energy piles," Geotechnique, vol. 68, no. 9, pp. 834-841, 2018, doi: 10.1680/jgeot.17.P.213.

[19] R. Saggu and T. Chakraborty, "Settlement Response of a Geothermal Energy Pile Group in Sand," in GeoChicago 2016, 2016, pp. 144-154.

[20] D. Pahud and M. Hubbuch, "Measured Thermal Performances of the Energy Pile System of the Dock Midfield at Zürich Airport," Procedding Eur. Geotherm. Congr. 2007, vol. 2, no. June, pp. 1-7, 2007.

[21] G. Kong, T. Cao, Y. Hao, Y. Zhou, and L. Ren, "Thermomechanical properties of an energy micro pile - raft foundation in silty clay," Undergr. Sp., 2019, doi: https://doi.org/10.1016/j.undsp.2019.09.005.

[22] M. Alberdi-Pagola, S. E. Poulsen, R. L. Jensen, and S. Madsen, "Thermal design method for multiple precast energy piles," Geothermics, vol. 78, pp. 201-210, 2019.

[23] C. Viggiani, Fondazioni. Benevento: Hevelius Edizioni, 1999.

[24] B. Das, Geotechnical Engineering Handbook. J. Ross Publishing, 2011.

[25] Presidenza del Consiglio dei Ministri, "Superbonus 110\%," 2020. http://www.governo.it/superbonus.

[26] F. Ronchi, D. Salciarini, N. Cavalagli, and C. Tamagnini, "Thermal response prediction of a prototype Energy Micro-Pile,” Geomech. Energy Environ., vol. 16, pp. 64-82, 2018, doi: 10.1016/j.gete.2018.07.001. 
[27] F. Ronchi, D. Salciarini, N. Cavalagli, and C. Tamagnini, "Numerical model of energy foundation behavior: the prototype of a geothermal micro-pile," Procedia Eng., vol. 158, pp. 326-331, 2016.

[28] R. Lautkankare, J. R. V. Sarola, and H. Kanerva-Lehto, "Hybrid energy micropiles in underpinning projectscombination of load bearing structures and geothermal energy field," 2014.

[29] M. Tyszer and B. Tomaszewska, "Evaluation of the Possibility of Use Geothermal Energy Micropiles TITAN 73/53 to Obtain Low-Temperature Heat Energy Accumulated in the Near-Surface Layers of the Ground in Poland Area," in Renewable Energy Sources: Engineering, Technology, Innovation, K. Mudryk and S. Werle, Eds. Springer International Publishing, 2017.

[30] L. wei Ren, J. Xu, G. qiang Kong, and H. long Liu, "Field tests on thermal response characteristics of microsteel-pipe pile under multiple temperature cycles," Renew. Energy, vol. 147, pp. 1098-1106, 2020, doi: 10.1016/j.renene.2019.09.084.

[31] A. Vieira et al., "Characterisation of ground thermal and thermo-mechanical behaviour for shallow geothermal energy applications," Energies, vol. 10, no. 12, 2017, doi: 10.3390/en10122044.

[32] B. Bezyan, S. Porkhial, and A. A. Mehrizi, "3-D simulation of heat transfer rate in geothermal pile-foundation heat exchangers with spiral pipe configuration," Appl. Therm. Eng., vol. 87, pp. 655-668, 2015.

[33] W. Yang, P. Lu, and Y. Chen, "Laboratory investigations of the thermal performance of an energy pile with spiral coil ground heat exchanger," Energy Build., vol. 128, pp. 491-502, 2016.

[34] A. Carotenuto, P. Marotta, N. Massarotti, A. Mauro, and G. Normino, "Energy piles for ground source heat pump applications: comparison of heat transfer performance for different design and operating parameters," Appl. Therm. Eng., vol. 124, pp. 1492-1504, 2017.

[35] J. Fadejev, R. Simson, J. Kurnitski, and F. Haghighat, “A review on energy piles design, sizing and modelling," Energy, vol. 122, pp. 390-407, 2017.

[36] S. Park, S. Lee, K. Oh, D. Kim, and H. Choi, "Engineering chart for thermal performance of cast-in-place energy pile considering thermal resistance," Appl. Therm. Eng., vol. 130, pp. 899-921, 2018.

[37] S. Park, S. Lee, D. Lee, D. Ahn, and H. Choi, "Effect of thermal interference on energy piles considering various configurations of heat exchangers," Energy Build., vol. 199, pp. 381-401, 2019.

[38] D. Salciarini and F. Cecinato, "Numerical Modelling of Thermo-active Micropiles," in International Conference of the International Association for Computer Methods and Advances in Geomechanics, 2021, pp. 1102-1109.

[39] R. W. Lewis, R. W. Lewis, and B. A. Schrefler, The finite element method in the static and dynamic deformation and consolidation of porous media. John Wiley \& Sons, 1998.

[40] S. W. Churchill, "Comprehensive correlating equations for heat, mass and momentum transfer in fully developed flow in smooth tubes," Ind. Eng. Chem. Fundam., vol. 16, no. 1, pp. 109-116, 1977.

[41] O. T. Farouki, "Thermal properties of soils," Cold Regions Research and Engineering Lab Hanover NH, 1981.

[42] S. W. Rees, M. H. Adjali, Z. Zhou, M. Davies, and H. R. Thomas, "Ground heat transfer effects on the thermal performance of earth-contact structures," Renew. Sustain. Energy Rev., vol. 4, no. 3, pp. 213-265, 2000.

[43] A. Ferrantelli, J. Fadejev, and J. Kurnitski, "Energy pile field simulation in large buildings: Validation of surface boundary assumptions," Energies, vol. 12, no. 5, pp. 1-20, 2019, doi: 10.3390/en12050770.

[44] M. Sutman, G. Speranza, A. Ferrari, P. Larrey-Lassalle, and L. Laloui, "Long-term performance and life cycle assessment of energy piles in three different climatic conditions," Renew. Energy, vol. 146, pp. 1177-1191, 2020, doi: 10.1016/j.renene.2019.07.035.

[45] Ai. Di Donna and L. Laloui, "Numerical analysis of the geotechnical behaviour of energy piles," Int. J. Numer. Anal. methods Geomech., vol. 39, no. 8, pp. 861-888, 2015.

[46] FHWA, "Micropile Design and Construction," 2005.

[47] S. B. Tatro, "Thermal properties," in Significance of tests and properties of concrete and concrete-making materials, ASTM International, 2006.

[48] R. Demirboğa, "Thermal conductivity and compressive strength of concrete incorporation with mineral admixtures," Build. Environ., vol. 42, no. 7, pp. 2467-2471, 2007.

[49] F. Girardi, G. M. Giannuzzi, D. Mazzei, V. Salomoni, C. Majorana, and R. Di Maggio, "Recycled additions for improving the thermal conductivity of concrete in preparing energy storage systems," Constr. Build. Mater., vol. 135, pp. 565-579, 2017, doi: https://doi.org/10.1016/j.conbuildmat.2016.12.179.

[50] K. Soga and Y. Rui, "Energy geostructures," in Advances in ground-source heat pump systems, Elsevier, 2016, pp. $185-221$.

[51] A. Ghozatloo, A. Rashidi, and M. Shariaty-Niassar, "Convective heat transfer enhancement of graphene nanofluids in shell and tube heat exchanger,” Exp. Therm. Fluid Sci., vol. 53, pp. 136-141, 2014.

[52] Y. Noorollahi, R. Saeidi, M. Mohammadi, A. Amiri, and M. Hosseinzadeh, "The effects of ground heat exchanger parameters changes on geothermal heat pump performance - A review," Appl. Therm. Eng., vol. 129, pp. 16451658, 2018, doi: 10.1016/j.applthermaleng.2017.10.111.

[53] O. Ogunleye, R. M. Singh, and F. Cecinato, "Assessing the thermal efficiency of energy tunnels using numerical methods and Taguchi statistical approach," Appl. Therm. Eng., vol. 185, p. 116377, 2021. 
[54] F. Loveridge and W. Powrie, "2D thermal resistance of pile heat exchangers," Geothermics, vol. 50, pp. 122135, 2014, doi: 10.1016/j.geothermics.2013.09.015.

[55] F. Loveridge, W. Powrie, and D. Nicholson, "Comparison of two different models for pile thermal response test interpretation," Acta Geotech., vol. 9, no. 3, pp. 367-384, 2014, doi: 10.1007/s11440-014-0306-3.

[56] D. Banks, An introduction to thermalgeology: Ground source heating and cooling. 2008.

[57] S. A. Bowers and R. J. Hanks, "Specific heat capacity of soils and minerals as determined with a radiation calorimeter," Soil Sci., vol. 94, no. 6, pp. 392-396, 1962, doi: 10.1097/00010694-196212000-00007.

[58] B. G. Clarke, A. Agab, and D. Nicholson, "Model specification to determine thermal conductivity of soils," Proc. Inst. Civ. Eng. Geotech. Eng., vol. 161, no. 3, pp. 161-168, 2008, doi: 10.1680/geng.2008.161.3.161.

[59] F. Cecinato, F. Loveridge, A. Gajo, and W. Powrie, "A new modelling approach for piled and other ground heat exchanger applications," in Geotechnical Engineering for Infrastructure and Development - Proceedings of the XVI European Conference on Soil Mechanics and Geotechnical Engineering, ECSMGE 2015, 2015, vol. 5.

[60] G. Taguchi and M. S. Phadke, "Quality engineering through design optimization," in Quality Control, Robust Design, and the Taguchi Method, Springer, 1989, pp. 77-96.

[61] G. S. Peace, Taguchi methods: a hands-on approach. Addison Wesley Publishing Company, 1993.

[62] F. Cecinato and A. Zervos, "Influence of thermomechanics in the catastrophic collapse of planar landslides," Can. Geotech. J., vol. 49, no. 2, 2012, doi: 10.1139/T11-095. 